\title{
Anomalous dimensions of four-fermion operators from conformal EWSB dynamics
}

\section{Luigi Del Debbio}

The Higgs Centre for Theoretical Physics

The University of Edinburgh, Edinburgh, UK

E-mail: Luigi.Del.Debbioded.ac.uk

\section{Liam Keegan}

Instituto de Física Teórica UAM/CSIC

Universidad Autónoma de Madrid, Cantoblanco 28049 Madrid, Spain*

E-mail: liam.keegan@cern.ch

\section{Carlos Pena ${ }^{\dagger}$}

Departamento de Física Teórica and Instituto de Física Teórica UAM/CSIC

Universidad Autónoma de Madrid, Cantoblanco 28049 Madrid, Spain

E-mail: carlos.pena@uam.es

\begin{abstract}
(Quasi)conformal scaling of composite operators from a strongly coupled EWSB dynamics helps to produce the characteristic hierarchies exhibited by the flavour couplings of the SM. It is however crucial to ensure that specific models satisfy bounds on Higgs and flavour dynamics; this in turn requires to control not only the anomalous dimensions of bilinears, but also those of higher-dimensional operators. We report on an ongoing effort to determine four-fermion operator anomalous dimensions, via Schrödinger Functional techniques, in the benchmark scenario of Minimal Walking Technicolour.
\end{abstract}

31st International Symposium on Lattice Field Theory - LATTICE 2013

July 29 - August 3, 2013

Mainz, Germany

\footnotetext{
*Address after November 1, 2013: CERN, Physics Department, 1211 Geneva 23, Switzerland
}

† Speaker. 


\section{Motivation}

One of the most interesting alternatives to Electroweak Symmetry Breaking (EWSB) by a weaklycoupled fundamental scalar field is to hypothetise the appearance of strong dynamics at or above the electroweak scale [1]. In order to be compatible with the tight constraints imposed by the flavour structure of the Standard Model, one particularly appealing possibility is that the symmetry breaking sector involves an (approximate) conformal symmetry that allows for a natural accommodation of flavour hierarchies in the dynamics, with the added possibility of having a light dilaton playing the role of the Higgs boson [3]. In recent years, various candidate field theories to display such a behaviour have been studied extensively on the lattice, using the sophisticated tools developed for QCD (see e.g. [4] for review at this conference).

One of the most important building blocks for model building in this context is the determination of the anomalous dimensions of composite operators. Apart from the obvious case of the techniquark mass anomalous dimension, it is also important to have information about four-fermion operators, that can have a relevant role not only in the flavour sector, but also e.g. in corrections to the Higgs couplings [5]. In this work we extend well-known QCD results on renormalisation of four-fermion operators to theories with adjoint fermion fields, and perform a preliminary study for the case of Minimal Walking Technicolour, building upon previous studies of this theory $[6,7]$.

\section{Renormalisation of four-fermion operators}

\subsection{Complete basis of operators}

The renormalisation of four-fermion operators in vector $\mathrm{SU}(N)$ gauge theories with fundamental fermion fields (e.g. QCD) is well understood. A comprehensive summary of the main results can be found e.g. in [8]. Generic Lorentz- and gauge-invariant operators can be written as

$$
\left(\bar{\psi}_{\alpha} \Gamma_{1} \psi_{\beta}\right)\left(\bar{\psi}_{\gamma} \Gamma_{2} \psi_{\delta}\right), \quad\left(\bar{\psi}_{\alpha} \Gamma_{1} T^{A} \psi_{\beta}\right)\left(\bar{\psi}_{\gamma} \Gamma_{2} T^{A} \psi_{\delta}\right)
$$

where $\alpha, \beta, \gamma, \delta$ are flavour indices; $\Gamma_{1,2}$ are spin matrices (possibly containing summed-over Lorentz indices); $T^{A}$ are colour generators with $A=1, \ldots, N^{2}-1$; and parentheses indicate traces over spin and colour.

In order to obtain a complete set of independent operators with a given flavour structure, one first observes that there are only ten independent spin structures $\Gamma_{1} \otimes \Gamma_{2}$, that can be furthermore chosen to lead to operators that transform in a well-defined way under parity:

$$
\begin{aligned}
& \text { parity-even: } \gamma_{\mu} \otimes \gamma_{\mu}, \gamma_{\mu} \gamma_{5} \otimes \gamma_{\mu} \gamma_{5}, \sigma_{\mu \nu} \otimes \sigma_{\mu \nu}, \mathbf{1} \otimes \mathbf{1}, \gamma_{5} \otimes \gamma_{5} ; \\
& \text { parity-odd: } \gamma_{\mu} \otimes \gamma_{\mu} \gamma_{5}, \gamma_{\mu} \gamma_{5} \otimes \gamma_{\mu}, \frac{1}{2} \varepsilon_{\mu v \rho \tau} \sigma_{\mu \nu} \otimes \sigma_{\rho \tau}, \mathbf{1} \otimes \gamma_{5}, \gamma_{5} \otimes \mathbf{1},
\end{aligned}
$$

where $\sigma_{\mu v}=\frac{i}{2}\left[\gamma_{\mu}, \gamma_{v}\right]$. Next, by using the colour trace identity and the spin Fierz identities in the particle-antiparticle channel

$$
\left(T^{A}\right)_{a b}\left(T^{A}\right)_{c d}=\frac{1}{2} \delta_{a d} \delta_{b c}-\frac{1}{2 N} \delta_{a b} \delta_{c d}, \quad\left(\Gamma_{1}^{(r)}\right)_{i j}\left(\Gamma_{2}^{(r)}\right)_{k l}=\sum_{s} f_{r s}\left(\Gamma_{1}^{(s)}\right)_{i l}\left(\Gamma_{2}^{(s)}\right)_{k j},
$$

where $f_{r s}$ are constants and the indices $r, s$ run over the ten spin structures above, one can rewrite operators containing colour generators as linear combinations of operators without colour generators (cf. Eq. (2.1)). The conclusion is that a complete basis of ten operators that are eigenstates of Fierz rearrangements with flavour content $(\alpha \beta \gamma \delta)$ is given by 


$$
\begin{aligned}
& Q_{1}^{ \pm} \equiv \frac{1}{2}\left[\left(\bar{\psi}_{\alpha} \gamma_{\mu}\left(\mathbf{1}-\gamma_{5}\right) \psi_{\beta}\right)\left(\bar{\psi}_{\gamma} \gamma_{\mu}\left(\mathbf{1}-\gamma_{5}\right) \psi_{\delta}\right) \pm(\beta \leftrightarrow \boldsymbol{\delta})\right], \\
& Q_{2}^{ \pm} \equiv \frac{1}{2}\left[\left(\bar{\psi}_{\alpha}\left(\mathbf{1}-\gamma_{5}\right) \psi_{\beta}\right)\left(\bar{\psi}_{\gamma}\left(\mathbf{1}+\gamma_{5}\right) \psi_{\delta}\right) \pm(\beta \leftrightarrow \boldsymbol{\delta})\right], \\
& Q_{3}^{ \pm} \equiv \frac{1}{2}\left[\left(\bar{\psi}_{\alpha} \gamma_{\mu}\left(\mathbf{1}-\gamma_{5}\right) \psi_{\beta}\right)\left(\bar{\psi}_{\gamma} \gamma_{\mu}\left(\mathbf{1}+\gamma_{5}\right) \psi_{\delta}\right) \pm(\beta \leftrightarrow \delta)\right], \\
& Q_{4}^{ \pm} \equiv \frac{1}{2}\left[\left(\bar{\psi}_{\alpha}\left(\mathbf{1}-\gamma_{5}\right) \psi_{\beta}\right)\left(\bar{\psi}_{\gamma}\left(\mathbf{1}-\gamma_{5}\right) \psi_{\delta}\right) \pm(\beta \leftrightarrow \delta)\right], \\
& Q_{5}^{ \pm} \equiv \frac{1}{2}\left[\left(\bar{\psi}_{\alpha} \sigma_{\mu v}\left(\mathbf{1}-\gamma_{5}\right) \psi_{\beta}\right)\left(\bar{\psi}_{\gamma} \sigma_{\mu v}\left(\mathbf{1}-\gamma_{5}\right) \psi_{\delta}\right) \pm(\beta \leftrightarrow \boldsymbol{\delta})\right],
\end{aligned}
$$

where $(\beta \leftrightarrow \delta)$ refers to a flavour exchange. It is customary to use a shorthand notation to make the spin structure and parity components explicit, viz. $Q_{1}^{ \pm}=Q_{\mathrm{VV}+\mathrm{AA}}^{ \pm}-Q_{\mathrm{VA}+\mathrm{AV}}^{ \pm} ; Q_{2}^{ \pm}=Q_{\mathrm{VV}-\mathrm{AA}}^{ \pm}+$ $Q_{\mathrm{VA}-\mathrm{AV}}^{ \pm} ; Q_{3}^{ \pm}=Q_{\mathrm{SS}-\mathrm{PP}}^{ \pm}+Q_{\mathrm{SP}-\mathrm{PS}}^{ \pm} ; Q_{4}^{ \pm}=Q_{\mathrm{SS}+\mathrm{PP}}^{ \pm}-Q_{\mathrm{SP}+\mathrm{PS}}^{ \pm} ; Q_{5}^{ \pm}=Q_{\mathrm{TT}}^{ \pm}+Q_{\mathrm{T} \overline{\mathrm{T}}}^{ \pm}$.

In order to carry over this result to the case of a theory with fermion fields transforming in the adjoint representation of the $\mathrm{SU}(N)$ colour group, the main difference lies in the structure of the colour trace identity and the corresponding spin Fierz rearrangement needed, that now takes place in the particle-particle channel,

$$
\left(T^{A}\right)_{a b}\left(T^{A}\right)_{c d}=\delta_{a d} \delta_{b c}-\delta_{a c} \delta_{b d}, \quad\left(\Gamma_{1}^{(r)}\right)_{i j}\left(\Gamma_{2}^{(r)}\right)_{k l}=\sum_{s} f_{r s}^{\prime}\left(\Gamma_{1}^{(s)} C\right)_{i k}\left(C \Gamma_{2}^{(s)}\right)_{l j},
$$

where $C$ is the charge conjugation matrix, that satisfies $C \gamma_{\mu} C^{T}=C^{T} \gamma_{\mu} C=-\gamma_{\mu}^{T}, C^{-1}=C^{T}$. In this way, operators of the form $\left(\bar{\psi}_{\alpha} \Gamma_{1} T^{A} \psi_{\beta}\right)\left(\bar{\psi}_{\gamma} \Gamma_{2} T^{A} \psi_{\delta}\right)$ can be written as linear combinations of operators of the forms $\left(\bar{\psi}_{\alpha} \Gamma_{1} \psi_{\delta}\right)\left(\bar{\psi}_{\gamma} \Gamma_{2} \psi_{\beta}\right)$ and $\left(\bar{\psi}_{\alpha} \Gamma_{1} C \bar{\psi}_{\gamma}^{T}\right)\left(\psi_{\delta}^{T} C \Gamma_{2} \psi_{\beta}\right)$.

As will be shown below, the occurrence of this second kind of operators with adjoint fermions does not lead to any qualitative difference with respect to the case of fundamental fermions, since the structure of the relevant correlation functions will be identical. Therefore, in the remainder of this section we will assume the structure in Eqs. (2.4-2.8).

\subsection{Operator mixing and anomalous dimensions}

Under renormalisation, any composite operator will mix with all operators of equal or lower engineering dimension that have the same transformation properties under all the relevant symmetries. In the case of mixing with lower-dimensional operators, the mixing coefficients can depend on fermion masses; this dependence can actually be constrained by treating the fermion mass matrix as a spurion field that transforms under the chiral group, such that the product of the operator times mass factors has the same transformation properties as the four-fermion operator of interest. The amount of flavour contained in the four-fermion operator plays a relevant role. For instance, the operator $\left(\bar{s}_{\mathrm{L}} \gamma_{\mu} d_{\mathrm{L}}\right)\left(\bar{s}_{\mathrm{L}} \gamma_{\mu} d_{\mathrm{L}}\right)$ that controls $\Delta S=2$ transitions in the Standard Model does not mix with lower-dimensional operators, since no operator of dimension lower than six can be written with two units of strangeness. On the opposite extreme, an operator of the form $\sum_{\alpha, \beta=1}^{N_{\mathrm{f}}}\left(\bar{\psi}_{\alpha} \psi_{\alpha}\right)\left(\bar{\psi}_{\beta} \psi_{\beta}\right)$, fully traced over flavour, will in general mix with several lower-dimensional operators.

The mixing structure is further complicated by the fact that regulators usually break some of the symmetries of the target renormalised theory; in particular, lattice regularisations break Poincaré symmetry and, unless Ginsparg-Wilson quarks are used, at least part of the full SU $\left(N_{\mathrm{f}}\right)_{\mathrm{L}} \times$ $\mathrm{SU}\left(N_{\mathrm{f}}\right)_{\mathrm{R}}$ chiral group. We will employ Wilson fermions, that break all axial chiral symmetries explicitly. This has two possible consequences: mixing with additional operators and less constrained mixing with lower-dimensional operators.

Mixing with lower-dimensional operators often leads to particularly complicated renormalisation problems. When the combined dimensions of the operator and the mass factors in the mixing coefficients add up to $d<6$ the missing powers of energy will lead to a power divergence $a^{d-6}$ in the bare four-fermion operator, that is also contained in the mixing coefficient such that the subtracted operator diverges only logarithmically. It can be shown that power-divergent subtractions 
do not contribute to anomalous dimensions [9]; as a matter of fact, if a mass-independent scheme is used renormalisation conditions can be imposed in the chiral limit, where subtractions that involve mass factors exactly vanish. When an operator requires subtractions that survive the chiral limit (as e.g. for the fully flavour-scalar operators mentioned above), it is possible to connect it via non-singlet chiral transformations (therefore keeping within the same chiral multiplet) to operators with enough flavour content so as to prevent mixing below $d=6$. It is thus convenient to extract anomalous dimensions from operators that do not require lower-dimensional subtractions.

A convenient way to achieve this is to consider operators that are made up by four formally distinct flavours, by setting $(\alpha \beta \gamma \delta)=(1234)$ in Eqs. (2.4-2.8). A full analysis of the resulting renormalisation problem is provided in [8]. First one can use the $\mathrm{SU}(4)_{\mathrm{L}} \times \mathrm{SU}(4)_{\mathrm{R}}$ chiral group spanned by the four flavours to determine operator mixing in the presence of full chiral symmetry; the outcome is that + and - operators do not mix, and that each of the two resulting five-operator sets displays the renormalisation pattern

$$
\left(\begin{array}{c}
\hat{Q}_{1}^{ \pm} \\
\hat{Q}_{2}^{ \pm} \\
\hat{Q}_{3}^{ \pm} \\
\hat{Q}_{4}^{ \pm} \\
\hat{Q}_{5}^{ \pm}
\end{array}\right)=\left(\begin{array}{ccccc}
Z_{11}^{ \pm} & 0 & 0 & 0 & 0 \\
0 & Z_{22}^{ \pm} & Z_{23}^{ \pm} & 0 & 0 \\
0 & Z_{32}^{ \pm} & Z_{33}^{ \pm} & 0 & 0 \\
0 & 0 & 0 & Z_{44}^{ \pm} & Z_{45}^{ \pm} \\
0 & 0 & 0 & Z_{54}^{ \pm} & Z_{55}^{ \pm}
\end{array}\right)\left(\begin{array}{c}
Q_{1}^{ \pm} \\
Q_{2}^{ \pm} \\
Q_{3}^{ \pm} \\
Q_{4}^{ \pm} \\
Q_{5}^{ \pm}
\end{array}\right)
$$

where $\hat{Q}_{i}^{ \pm}$denotes a renormalised operator. Note that, furthermore, each operator can be split into even and odd pieces under parity; operators with different parity do not mix, and they renormalise with the same matrix of renormalisation constants. With Wilson fermions, the breaking of chiral symmetry leads to all operators mixing; using an obvious vector notation, this can be written as

$$
\hat{\vec{Q}}^{ \pm}=Z^{ \pm}\left(\mathbf{1}+\Delta^{ \pm}\right) \vec{Q}^{ \pm}
$$

where $\Delta^{ \pm}$are matrices that have non-zero entries where $Z^{ \pm}$has zero entries, and vice versa. Its elements $\Delta_{i j}^{ \pm}$do not depend on the renormalisation scale, but only on the bare lattice coupling. The structure of $\Delta^{ \pm}$can be further constrained by exploiting discrete symmetries, including the discrete subgroup of flavour exchange symmetries that are left intact by a Wilson term. One crucial result is that parity-odd operators are protected by the so-called CPS symmetries, that combine charge conjugation, parity, and various flavour switchings. It then follows that, if Eq. (2.11) is projected into well-defined parity sectors, then $\Delta_{i j}^{ \pm}=0$ for odd parity - i.e. the set of operators $Q_{\mathrm{VA}+\mathrm{AV}}^{ \pm}, \ldots, Q_{\mathrm{T} \tilde{\mathrm{T}}}^{ \pm}$renormalises as in Eq. (2.10) even with Wilson fermions. Since the continuum anomalous dimensions are the same for the parity-even and parity-odd parts of the basis, the final conclusion is that the cleanest way to determine them is to impose renormalisation conditions on parity-odd, fully-flavoured operators in the chiral limit.

\section{Non-perturbative computation of anomalous dimensions}

Our setup to determine the anomalous dimensions of four-fermion operators in theories with adjoint fermions follows closely the approach of [10], where anomalous dimensions of four-fermion operators in QCD were studied exploiting the Schrödinger Functional (SF) formalism. The theory is regularised on a lattice of physical size $L^{4}$ with standard SF Dirichlet boundary conditions, that allow to simulate at vanishing fermion mass, and periodic boundary conditions for fermions in space up to a phase $\theta$. Renormalisation conditions are imposed at a scale $\mu=1 / L$. To define them one first introduces SF correlation functions of the form 


$$
F_{i ; \mathrm{A}, \mathrm{B}, \mathrm{C}}^{ \pm}\left(x_{0}\right)=\frac{1}{L^{3}}\left\langle\mathscr{O}_{53}^{\prime}\left[\Gamma_{\mathrm{C}}\right] \mathscr{Q}_{i}^{ \pm} \mathscr{O}_{21}\left[\Gamma_{\mathrm{A}}\right] \mathscr{O}_{45}\left[\Gamma_{\mathrm{B}}\right]\right\rangle
$$

where $\mathscr{Q}_{i}^{ \pm}$is the parity-odd part of $Q_{i}^{ \pm} ; \mathscr{O}, \mathscr{O}^{\prime}$ are interpolating fields on the time boundaries; and five different choices for the Dirac matrices $\Gamma_{\text {A.B.C }}$ are possible - see [10] for full details. Note that a fifth "spectator" flavour has been introduced. ${ }^{1}$ The logarithmic divergence of $\mathscr{Q}_{i}^{ \pm}$can be isolated by dividing out the divergencies coming from the boundaries, through ratios of the form

$$
h_{i ; \mathrm{A}, \mathrm{B}, \mathrm{C}}^{ \pm}\left(x_{0}\right)=\frac{F_{i ; \mathrm{A}, \mathrm{B}, \mathrm{C}}^{ \pm}\left(x_{0}\right)}{f_{1}^{\eta} k_{1}^{3 / 2-\eta}},
$$

where $f_{1}=-1 /\left(2 L^{6}\right)\left\langle\mathscr{O}_{21}^{\prime}\left[\gamma_{5}\right] \mathscr{O}_{12}\left[\gamma_{5}\right]\right\rangle$ and $k_{1}=-1 /\left(6 L^{6}\right)\left\langle\mathscr{O}_{21}^{\prime}\left[\gamma_{k}\right] \mathscr{O}_{12}\left[\gamma_{k}\right]\right\rangle$ are boundary-to-boundary correlators, and the value of $\eta \in[0,3 / 2]$ is fixed by the choice of $\Gamma_{\mathrm{A}, \mathrm{B}, \mathrm{C}}$.

Renormalisation conditions for the multiplicatively renormalisable operators $\mathscr{Q}_{1}^{ \pm}=Q_{\mathrm{VA}+\mathrm{AV}}^{ \pm}$ have the form

$$
Z_{1}^{ \pm}\left(g_{0}, a \mu\right) h_{1 ; \mathrm{A}, \mathrm{B}, \mathrm{C}}^{ \pm}(L / 2)=\left.h_{1 ; \mathrm{A}, \mathrm{B}, \mathrm{C}}^{ \pm}(L / 2)\right|_{g_{0}=0} .
$$

For the other operators, that mix in doublets, one has to impose matrix renormalisation conditions of the form

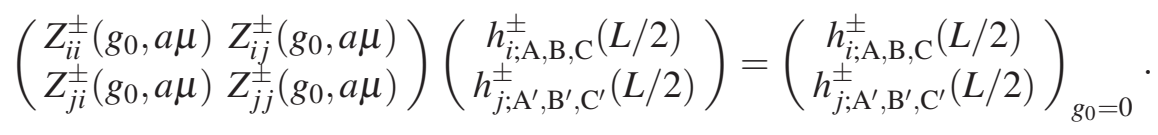

Different choices of boundary matrices result in different renormalisation schemes. In either case, sensible sets of renormalisation conditions require that $Z^{ \pm}$is well-defined and equal to the identity at tree-level.

The equivalence of the formally different fierzings that appear in theories with fundamental and adjoint fermions, alluded to above, can be easily appreciated by working out the Wick contractions that enter the correlation functions $h_{i ; \mathrm{A}, \mathrm{B}, \mathrm{C}}^{ \pm}$in either case. Let us substitute $\mathscr{Q}_{i}^{ \pm}$in Eq. (3.2) for either $\left(\bar{\psi}_{1} \Gamma_{1} \psi_{2}\right)\left(\bar{\psi}_{3} \Gamma_{2} \psi_{4}\right),\left(\bar{\psi}_{1} \Gamma_{1} \psi_{4}\right)\left(\bar{\psi}_{3} \Gamma_{2} \psi_{2}\right)$, or $\left(\bar{\psi}_{1} \Gamma_{1} C \bar{\psi}_{3}^{T}\right)\left(\psi_{4}^{T} C \Gamma_{2} \psi_{2}\right)$. After integration over fermion fields, one has for the respective Wick contractions expressions of the form

$$
\begin{aligned}
& \left\langle\operatorname{Tr}\left\{H_{1}(x)^{\dagger} \gamma_{5} \Gamma_{1} H_{2}(x) \Gamma_{\mathrm{A}} \gamma_{5}\right\} \operatorname{Tr}\left\{H_{3}^{\prime}(x)^{\dagger} \gamma_{5} \Gamma_{2} H_{4}(x) \Gamma_{\mathrm{B}} \gamma_{5} \mathscr{H}_{5}^{\dagger} \Gamma_{\mathrm{C}} \gamma_{5}\right\}\right\rangle_{\mathrm{G}}, \\
- & \left\langle\operatorname{Tr}\left\{H_{1}(x)^{\dagger} \gamma_{5} \Gamma_{1} H_{4}(x) \Gamma_{\mathrm{B}} \gamma_{5} \mathscr{H}_{5}^{\dagger} \Gamma_{\mathrm{C}} \gamma_{5} H_{3}^{\prime}(x)^{\dagger} \gamma_{5} \Gamma_{2} H_{2}(x) \Gamma_{\mathrm{A}} \gamma_{5}\right\}\right\rangle_{\mathrm{G}}, \\
- & \left\langle\operatorname{Tr}\left\{H_{1}(x)^{\dagger} \gamma_{5} \Gamma_{1} H_{\tilde{4}}(x) \Gamma_{\mathrm{B}} \gamma_{5} \mathscr{H}_{\tilde{5}}^{\dagger} \Gamma_{\mathrm{C}} \gamma_{5} H_{\tilde{3}}^{\prime}(x)^{\dagger} \gamma_{5} \Gamma_{2} H_{2}(x) \Gamma_{\mathrm{A}} \gamma_{5}\right\}\right\rangle_{\mathrm{G}}
\end{aligned}
$$

where $H, H^{\prime}$ are bulk-to-boundary fermion propagators, $\mathscr{H}$ is the boundary-to-boundary fermion propagator, and, in the last expression, propagators bearing a tilde in the flavour index are those for the fermion variables $\tilde{\psi}(x) \equiv C \bar{\psi}(x)^{T}, \overline{\tilde{\psi}}(x) \equiv \psi(x)^{T} C$. It is thus obvious that the structure of the correlation functions are identical for either fermion representation.

Using standard finite size-scaling techniques, it is possible to compute the renormalisation group running of the operators by computing renormalisation constants on several lattices tuned so that the continuum limit can be taken at fixed $\mu=1 / L$ for several values of $\mu$, using a previous determination of the SF running coupling $\bar{g}(\mu)$. The basic objects to that purpose are the so-called step-scaling functions (ssf)

\footnotetext{
${ }^{1}$ In case the theory of interest has less than five dynamical flavours, this setup can be interpreted in terms of a valence sector used to the sole purpose of imposing renormalisation conditions. Since all computations are carried out in the chiral limit, there is no subtlety related to the matching of valence and sea fermions.
} 

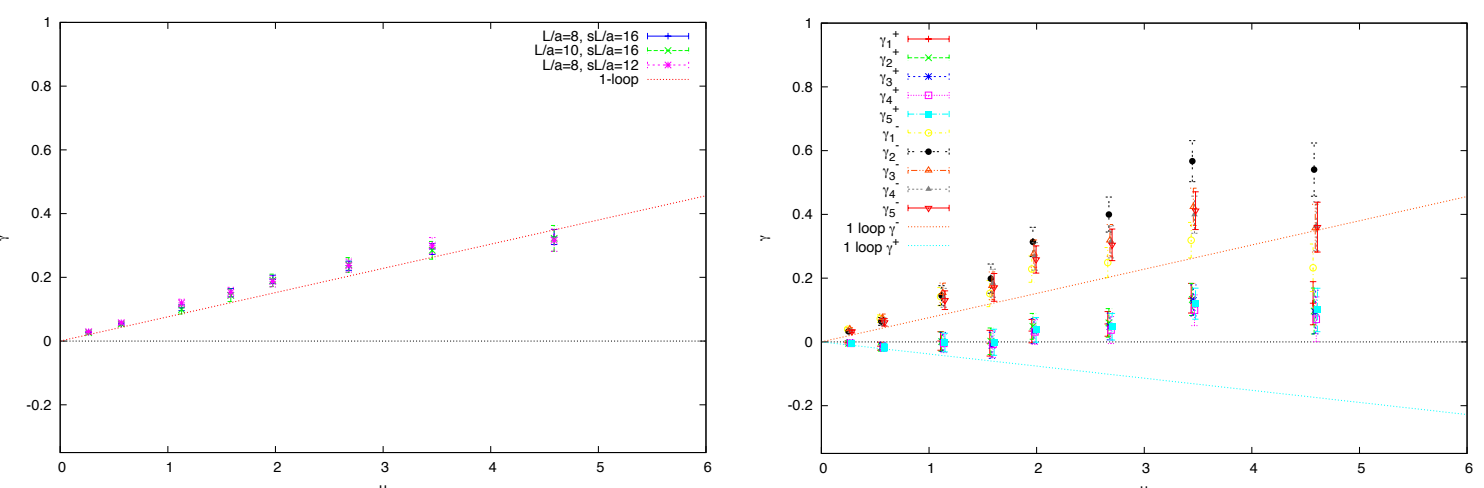

Figure 1: Left: anomalous mass dimension for various scaling steps. Right: anomalous dimensions of $Q_{\mathrm{VA}+\mathrm{AV}}^{ \pm}$from $L / a=8 \rightarrow 16$.
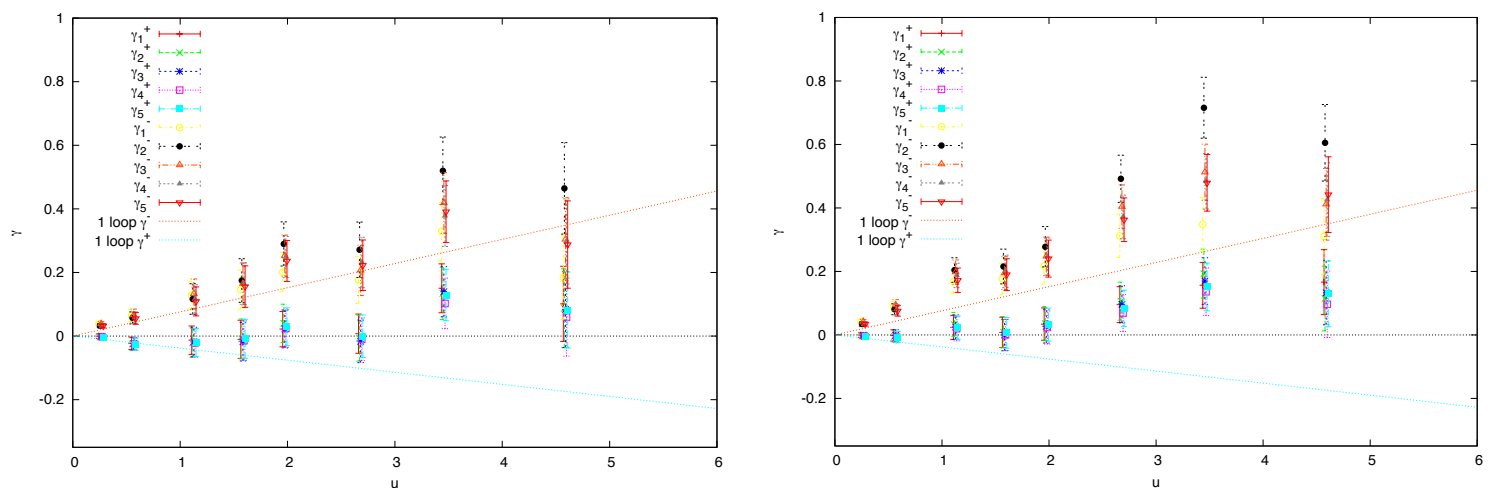

Figure 2: Left: anomalous dimensions of $Q_{\mathrm{VA}+\mathrm{AV}}^{ \pm}$from $L / a=10 \rightarrow 16$. Right: idem from $L / a=8 \rightarrow 12$.

$$
\left.\Sigma^{ \pm}(s ; u, a / L) \equiv Z^{ \pm}\left(g_{0}, a \mu / s\right)\left[Z^{ \pm}\left(g_{0}, a \mu\right)\right]^{-1}\right|_{u=\bar{g}^{2}(\mu)},
$$

where $s>1$ is some fixed scaling parameter, that can be defined either for multiplicatively renormalisable operators or for operator doublets. The continuum limit of an ssf can be easily written in terms of the continuum anomalous dimension matrix $\gamma^{ \pm}$and the $\beta$ function as

$$
\sigma^{ \pm}(s ; u) \equiv \lim _{a \rightarrow 0} \Sigma^{ \pm}(s ; u, a / L)=\operatorname{Texp}\left\{\int_{\bar{g}(\mu)}^{\bar{g}(\mu / s)} \mathrm{d} g \frac{\gamma^{ \pm}(g)}{\beta(g)}\right\},
$$

where the Texp is meant to be ordered in $g$. Close to an infrared fixed point, a good approximant for the anomalous dimension is $\gamma^{ \pm}(\bar{g}(\mu)) \approx\left[\log \sigma^{ \pm}(s ; u)\right] /[\log s]$.

\section{Preliminary results in MWTC}

Minimal Walking Technicolour - i.e. an SU(2) gauge theory with two flavours of fermions transforming in the adjoint colour representation - has been simulated at vanishing PCAC quark mass using the Hybrid MonteCarlo algorithm for lattices of size $L / a=8,10,12,16$ and $\beta=$ $16.00,8.00,4.50,3.50,3.00,2.50,2.20,2.05$ for each lattice, using the Wilson plaquette action and the unimproved Wilson fermion action. SF correlation functions for four-fermion operators, as well as quark bilinears (which allows to study the running techniquark mass, providing a cross-check with and extending the study of [6]), have been computed at vanishing background field and a value of $\theta=0.5$ for the abelian spatial twist. Approximately 20000 thermalised trajectories are available at each simulation point, with acceptance rates ranging between $83 \%$ and $96 \%$.

The left panel of Fig. 1 shows (minus) the quantity $\log \sigma(s ; u) / \log s$ for the pseudoscalar density (i.e. the mass anomalous dimension) for various step scalings. The right panel shows the 
same quantity for the multiplicatively renormalisable operators $Q_{\mathrm{VA}+\mathrm{AV}}^{ \pm}$and fixed step scaling from $L / a=8$ to $L / a=16$. In the latter case the five available renormalisation schemes are shown. The values of $\beta$ have been converted to values of $u=\bar{g}^{2}$ based on the running coupling data at $L=8$, and the one-loop predictions are also shown on the plots for comparison. While the results for the four-fermion anomalous dimensions are much less precise, owing to the use of four-point correlation functions and the intrinsically noisier four-fermion insertion, they display a remarkable independence of the scheme choice, as expected for a theory that displays conformal behaviour and quite contrary to the findings in QCD [10]. Within the large errors, around the values of the coupling for which the appearance of the fixed point is suspected one has $\gamma^{+} \sim 0, \gamma^{-} \sim 0.4$. The mild dependence on the choice of the step scaling is illustrated in Fig. 2, which confirms the rough consistence with the expectations coming from conformal dynamics. Preliminary results for the anomalous dimension matrices involving the other four operators, which include the phenomenologically interesting case $S P+P S$, are also consistent with this picture.

\section{Outlook}

Studying the anomalous dimensions of four-fermion operators is an important step in the understanding the phenomenological implications of strongly coupled models for EWSB. We have reviewed the general framework to determine anomalous dimensions non-perturbatively, and extended it to theories with adjoint fermions.

Our MWTC results are quite preliminary, not least because of the uncontrolled systematic uncertainties stemming from not having a continuum limit, a significant statistical error, and our uncertain knowledge about the location of the (possible) fixed point. Yet, our results show a nontrivial consistency with expectations from conformal dynamics, and provide ballpark values for the anomalous dimensions in the would-be conformal region. After our analysis is complete, including results for the full basis of operators, a next obvious step is to exploit the ample margin for better precision offered by increasing our statistics, simulating larger lattices to allow for a better study of the approach to the continuum limit, or implementing $\mathscr{O}(a)$ improvement. Extension to other models with adjoint or fundamental fermions is straightforward.

\section{References}

[1] S. Weinberg, Phys. Rev. D 19 (1979) 1277; L. Susskind, Phys. Rev. D 20 (1979) 2619.

[2] B. Holdom, Phys. Rev. D 24 (1981) 1441.

[3] K. Yamawaki, M. Bando and K.-I. Matumoto, Phys. Rev. Lett. 56 (1986) 1335; T. Akiba and T. Yanagida, Phys. Lett. B 169 (1986) 432; T.W. Appelquist, D. Karabali and L.C.R. Wijewardhana, Phys. Rev. Lett. 57 (1986) 957.

[4] J. Kuti, these proceedings.

[5] R. Rattazzi, V. S. Rychkov, E. Tonni and A. Vichi, JHEP 0812 (2008) 031.

[6] F. Bursa et al., Phys. Rev. D 81, 014505 (2010).

[7] L. Del Debbio et al., Phys. Rev. D 82, 014509 (2010); L. Del Debbio et al., Phys. Rev. D 82, 014510 (2010); T. DeGrand, Y. Shamir and B. Svetitsky, Phys. Rev. D 83, 074507 (2011); S. Catterall, L. Del Debbio, J. Giedt and L. Keegan, Phys. Rev. D 85, 094501 (2012).

[8] A. Donini et al., Eur. Phys. J. C 10 (1999) 121

[9] M. Testa, JHEP 9804 (1998) 002.

[10] M. Guagnelli et al. [ALPHA Collaboration], JHEP 0603 (2006) 088; F. Palombi, C. Pena and S. Sint, JHEP 0603 (2006) 089; P. Dimopoulos et al. [ALPHA Collaboration], JHEP 0805 (2008) 065. 